\title{
Safety of Cow's Milk-Derived Fortifiers Used with an All-Human Milk Base Diet in Very Low Birthweight Preterm Infants
}

Alan Lucas MD FMedSci, Maushumi Assad MD, MPH, Jan Sherman PhD, John Boscardin PhD, Steven Abrams MD

\begin{abstract}
Background: Very low birthweight (VLBW) preterm infants fed mothers own milk (MOM) need nutritional supplementation, traditionally achieved with cow's milk (CM) derived fortifier CMDF) and preterm formula (PTF) if MOM is insufficient. CM products have been associated with diverse major morbidities. The current recommendation is to preferentially replace PTF with donor milk (DM) to produce a 100\% human milk (HM) base diet, usually fortified with CMDF. Objective: To identify whether CMDF, even when fed with a $100 \%$ HM base diet, is related to an increased risk of major morbidities.
\end{abstract}

Methods: We identified a randomized trial with an all-HM base diet, comparing CMDF with a fortifier derived from human milk (HMDF), and two additional studies of this design were generated from raw data as subgroup analyses of a randomized controlled trial and a quasi-experimental study. Using these studies, we calculated the impact of CMDF on major morbidities of death, necrotizing enterocolitis (NEC), retinopathy of prematurity (ROP), sepsis, bronchopulmonary dysplasia (BPD) and patent ductus arteriosus (PDA).

Results: Each study individually provided support for an increase in major morbidities with CMDF. Meta-analyses of pooled data showed that compared to HMDF, the CMDF group had large increases in NEC (RR=3.3; $P=0.001), R O P(R R=2.2 ; P=0.007)$, $P D A \quad(R R=1.6 ; \quad P=0.009)$, interruption of feeding $(R R=3.4$; $P=0.001)$ and a positive mortality/morbidity index based on one or more of death, NEC, sepsis, ROP and BPD (RR=1.4; $P=0.006)$.

Conclusions: Despite the increased use of HM in modern neonatal care as a base diet, we found a greater risk of critical morbidities with CMDF compared with HMDF. This burden of morbidity provides evidence that the benefits of an HM base diet, might be, in part, counteracted by multiple adverse outcomes relating to the use of CMDF.

Key Words: preterm infant feeding, cow's milk-derived fortifiers, human milk-derived fortifiers, neonatal morbidity, donor milk

Abbreviations: MOM: Mothers own milk, CM: Cow's milk, CMDF: Cow's milk-derived fortifier, PTF: Preterm fortifier, HMDF: human milk-derived fortifier, NEC: Necrotizing enterocolitis, ROP: Retinopathy of prematurity, PDA: Patent ductus arteriosis, BPD: bronchopulmonary dysplasia, NICU: Neonatal intensive care unit

\section{Introduction}

MOM is strongly recommended for very low birth weight (VLBW) preterm infants, but does not fully meet their nutritional needs, traditionally met by adding a CMDF to MOM, and when MOM is insufficient, by using a PTF. CM-based products have an important role in current neonatal practice in promoting growth, but evidence indicates that VLBW infants fed partly or wholly on CM products may have a greater risk of adverse outcomes relating to NEC14, late-onset sepsis (LOS) (5-8), mortality $(7,8)$, ROP $(7,9-11)$, BPD, $(10,12)$, brain development $(13,14)$, cardiovascular risk (15$17)$, bone health (18), atopic disease (19) and structural development of the heart, lungs and great vessels (20). It is unknown if these adverse outcomes relate to $\mathrm{CM}$ exposure, displacement of HM exposure, or both.

With increasing focus on using human milk in the NICU, official bodies $(21,22)$ recommend using DM rather than PTF when MOM is insufficient, thus increasing HM exposure. Most units would then use a CMDF as the sole source of CM. Given the international emergence of this practice, testing the safety of CMDF, as used in this common practice, is critical. The ideal safety study is one where the base diet is $100 \% \mathrm{HM}$, and where it is possible to compare a CMDF versus an HMDF for a range of morbidities. However, remarkably few such studies have been undertaken. We identified only three studies of this design; the OptiMoM trial (9) together with two subgroup analyses of existing studies $(1,10)$.

\section{"Our hypothesis, based on historical evidence of adverse outcomes seen with CM products (cited above), is that even when the base diet is $100 \%$ HM, CMDF is associated with major morbidity."}

Our hypothesis, based on historical evidence of adverse outcomes seen with CM products (cited above), is that even when the base diet is $100 \% \mathrm{HM}, \mathrm{CMDF}$ is associated with major morbidity. A large, well-powered, hypothesis-testing trial has not been done to test this comprehensively. However, since feeding an all $\mathrm{HM}$ diet with a CMDF is so prevalent, we considered that the combined analysis of morbid outcomes and mortality from the three studies identified, providing 453 subjects, should be evaluated as this is the largest dataset of its type and may help to guide practice and research.

\section{Methods}

Screening of PUBMED, MEDLINE, Google Scholar, and recent reviews, revealed only one study, the OptiMoM trial (2018) (9),

NEONATOLOGY TODAY is interested in publishing manuscripts from Neonatologists, Fellows, NNPs and those involved in caring for neonates on case studies, research results, hospital news, meeting announcements, and other pertinent topics.

Please submit your manuscript to: LomaLindaPublishingCompany@gmail.com 
that met our criterion of $100 \%$ human milk base diet with the experimental comparison of CMDF with a modern HMDF. However, we reanalyzed part of this study, and the authors later corrected the findings (see results). The other two studies - the Sullivan trial 2010 (1) and the Assad study 2014 (10) - were ones where we had access to raw data and could generate a subgroup with the desired design (100\% HM base diet and experimental comparison of CMDF versus HMDF). We rejected a group of very small studies, notably those of Polberger et al (23) and Hagelberg et al (24) from before the modern HMDF era where investigative groups made non-standardized, clinically unavailable fortifier preparations from skimmed DM for physiological studies that were experimental precursors of current standardized HMDFs.

The three studies presented here are considered separately; and then combined in meta-analyses if two or all three studies included major individual morbidities, previously linked to CM exposure, including NEC, death, ROP, BPD, LOS, and PDA. A secondary outcome was feeding interruption (enteral feeding withheld 24 hours; FW24) since the increased need for parenteral nutrition (PN) with feed intolerance may increase morbidity.

\section{"The three studies presented here are considered separately; and then combined in meta-analyses if two or all three studies included major individual morbidities, previously linked to CM exposure, including NEC, death, ROP, $B P D$, LOS, and PDA."}

The original studies included here for further reanalyses and meta-analyses all received ethical approval. The OptiMoM trial was approved by the Human Research Ethics Board of each participating hospital; for the trial by Sullivan et al (2) Institutional Review Board (IRB) approval was obtained at each center; and for the Assad, study (3) approval was obtained from the IRB of the Herman and Walter Samuelson Children's Hospital.

\section{OptiMoM trial (9)}

This was a blinded, multicenter RCT from Canada comprising 125 infants $<1250 \mathrm{~g}$ birthweight, who received a base diet of MOM, plus DM as required, and were randomly assigned to CMDF or HMDF. The trial was powered for feeding interruption, but a secondary outcome was a dichotomous mortality/morbidity index, affirmative for any one or more of death, LOS, BPD, ROP or NEC (Bells stage II or greater) - each reported separately as well as part of the morbidity/mortality index. Partial BM fortification commenced at a feeding volume of $100 \mathrm{ml} / \mathrm{kg}$ per day and full fortification at $140 \mathrm{ml} / \mathrm{kg} /$ day.

\section{Sullivan trial; subgroup analysis}

The original trial (1), reported elsewhere, was a 3-limb, 12 center RCT (11 centers from the USA: one from Austria) including infants $500-1250 \mathrm{~g}$ at birth. All infants received MOM. Groups HM100 and HM40 received HMDF (Prolacta Bioscience, USA), at feeding volumes of $100 \mathrm{~mL} / \mathrm{kg} / \mathrm{day}$ and $40 \mathrm{~mL} / \mathrm{kg} / \mathrm{day}$, and both groups received standardized DM (Prolacta Bioscience) when MOM was insufficient. The CM group received CMDF (Enfamil, Mead Johnson, USA or Similac; Abbott Laboratories, USA) and a PTF if MOM insufficient.
We have recently reported 25 an analysis of a subgroup from that trial in which the base milk was $100 \%$ mother's milk. Thus, the only difference between groups was whether HMDF or CMDF was added. Those randomized separately into the HM40 and HM100 were merged for analysis (no differences were seen based on the timing of initiation of feeds for any outcome), explaining why there were 82 subjects fed HMDF and 32 fed CMDF.

NEC was the principal outcome in our subgroup reanalysis, justified by the fact that it was a predetermined key outcome in the main trial (Clinicaltrials.gov reg. \#NCT00506584.). Our further key outcome was a severe morbidity index: NEC surgery or death. Because mortality is high in those requiring surgery, death is logically included because of its censoring effect on the incidence of NEC surgery. Secondary outcomes included: BPD $(7,10-12)$, ROP7,9-11, and sepsis (5-8).

\section{Assad subgroup analysis}

Assad et al. (2014) (10) conducted a single-center study in 293 preterm infants (mean gestation 28 weeks). The study is reported elsewhere (10). Three groups of infants were fed CM-based products and a 4th, EHM feeding. Two of the groups allowed a pre-post (quasi-experimental) comparison of an all HM base diet with CMDF pre-2012 versus an all HM base diet with HMDF post2012. Since some original data analyses were from combined groups, our new 2-subgroup study required a reanalysis of primary data, allowing us to examine any association between fortifier type and multiple outcomes.

\section{Publication history}

The data in the Assad subgroup reanalysis, have not been published previously as an original manuscript. This analysis now also includes new data from the medical records for death and LOS that were not included in the analysis of the original Assad study (10). Both the Sullivan reanalysis and OptiMoM studies have been published $(9,25)$ but have been further analyzed in this current study. None of the meta-analyses of the three studies combined have been previously published.

\section{Type of human milk}

The exclusive human milk group in the three studies all included both MOM and donor milk-derived fortifier. We note that in two of the three studies (Sullivan and Assad), the base diet in the CM group was only MOM, whereas in the OptiMoM trial, the base diet was MOM plus DM as required.

\section{Diagnosis of NEC}

In the OptiMoM and Sullivan trials, the diagnosis of NEC was made by radiologists, blind to the dietary assignment to improve diagnostic reliability, as described previously (2).

\section{ROP}

Two studies examined severe ROP (Sullivan(1) and OptiMoM(9)), and one examined all ROP (Assad10). For our meta-analyses, we elected to combine the three studies despite the heterogeneity. Previous studies have done this, and prior work shows both severe and all ROP are related to the use of $\mathrm{CM}(11)$ - as seen here when the studies were analyzed separately.

\section{Statistical analysis}

The baseline comparisons of categorical data used the chi-square test for homogeneity or Fisher's exact test for small cell sizes. Comparisons of baseline quantitative variables used the two-sample t-test. In considering whether fixed or random-effects models 
would be most appropriate for our meta-analyses, we note the view of Borenstein et al (26). that if the number of studies is very small, as in this case, it may be impossible to estimate adequately between-study variance (tau-squared), rendering the fixed effect model the most viable option. In effect, we have treated included studies as the only ones of interest. Nevertheless, for the more major analyses, we also checked whether similar findings emerged with a random-effects model. We performed meta-analyses using REVMAN 5.3 software.

\section{Results}

Table 1 shows a lack of statistical evidence for differences in baseline characteristics between CMDF and HMDF groups within any of the three studies ( $P>0.05$ in all cases).

Table 2 shows the relationship between fortifier type and outcomes for the three individual studies.

\section{OptiMoM trial (9)}

For all outcomes shown for OptiMoM in table 2, RR was greater in the CMDF group ranging from negligible for NEC stage II or greater, to 6.4 for severe ROP, a significant effect $(P=0.04)$. There was also evidence for higher risk of sepsis with CMDF $(R R=1.84$; that is, an $84 \%$ increase in risk: $P=0.07$ ).

Additionally, the authors reported a dichotomous combined overall morbidity index, which was positive if the subject exhibited one or more of the following: death, NEC (stage II or higher), BPD, ROP or LOS. This aspect of the trial results is material, yet historically complex and clarified here,

The initial publication of the trial findings showed this morbidity index was not related to fortifier type: the incidence of a positive index in the CMDF and HMDF groups was almost identical at $49 \%$ and $48 \%$. (9)

\section{"However, we were concerned that} this analysis was not an accurate representation of the data. Our own analysis utilized the authors' data on individual morbidities to calculate the average number of adverse events per subject. There were 31 adverse events in 64 subjects fed HMDF (0.48 events per subject); yet, for CMDF there were 45 adverse events among 61 subjects $(0.74$ events per subject)."

However, we were concerned that this analysis was not an accurate representation of the data. Our own analysis utilized the authors' data on individual morbidities to calculate the average number of adverse events per subject. There were 31 adverse events in 64 subjects fed HMDF ( 0.48 events per subject); yet, for CMDF there were 45 adverse events among 61 subjects $(0.74$ events per subject). Raw data were unavailable, but we did a provisional analysis of the overall morbidity data reported using a large sample z-test approximation to a Poisson distribution. The significance level was $\mathrm{P}=0.06$ for the ratio of adverse events per case in the CMDF vs. HMDF group. The point estimate was an $\mathrm{RR}$ of 1.50 (95\% Cl: 0.95 to 2.37 ), that is, a $50 \%$ higher risk of a positive index in the CMDF group. Our analysis, while limited and hence provisional, nevertheless suggested that the CMDF group might prove disadvantaged for multiple morbidities.

The authors of the OptiMoM trial re-explored their findings and published a corrigendum (27), noting that there had been an overcounting of BPD cases resulting in their removal of 8 cases with a positive index selectively from the HMDF group. Thus, a revised version of the OptiMoM trial results now shows 23/64 subjects with a positive mortality/morbidity index in the HMDF group and $30 / 61$ subjects in the CMDF group, reflecting, in our own analysis, an increased RR of having a positive index in the CMDF group of approaching $40 \%$ (RR $1.37 ; 95 \% \mathrm{Cl} 0.90-2.07$ ) with an authors' adjusted $\mathrm{P}=0.07$. The revised version is the version of the record at the journal website (direct link to AJCN).

\section{Sullivan trial subgroup analysis (25)}

Table 2 shows that use of CMDF was related to an increase in NEC compared to use of HMDF ( $R R=4.2 ; P=0.04)$; and also to a severe morbidity index of NEC surgery or death $(R R=5.1 ; P=0.01)$ For BPD, sepsis, and ROP the relationship with fortifier type was not found.

\section{Assad study reanalysis}

The quasi-experimental pre-post subgroup analysis of Assad's study showed a major impact of fortifier type in those otherwise fed $100 \%$ human milk base diet.

Table 2 shows 7 outcomes in relation to fortifier type: death, lateonset sepsis, BPD, NEC, ROP, PDA, and FW24, all of which showed at an increase in risk in the CMDF group, and significantly so for NEC (RR=7.5:P=0.02); ROP $(R R=2.5 ; P=0.001)$; $P D A$ $(R R=2.7 ; P=0.007) ; F W 24(R R=5.9 ; P=0.001)$.

In the original 4-limb Assad study, BPD incidence was significantly higher in those exposed to CM, but this subgroup analysis that allowed us to compare fortifiers was underpowered to study BPD; nevertheless, there was a $60 \%$ higher risk of BPD in the CMDF group $(\mathrm{RR}=1.6)$.

\section{Meta-analyses}

All three studies, Assad, Sullivan, and OptiMoM, contributed data for our meta-analyses of NEC, ROP. Death, BPD, and sepsis (all shown in Fig 1). Using fixed effect models, as planned, CMDF was associated with a higher risk of NEC ( $R R=3.3 ; P=0.008)$ and $\mathrm{ROP}(\mathrm{RR}=2.4 ; \mathrm{P}=0.001)$; with significance also shown in randomeffects models (not depicted). In the CMDF group, there was also a more than doubling of the risk of death $(R R=2.1 ; P=0.1)$; and a $32 \%$ higher risk of $B P D(R R=1.32 ; P=0.1)$. Both effects trended towards statistical significance. No overall effect of fortifier type was found for late-onset sepsis: the $80 \%$ and $30 \%$ increases in risk with CMDI in the OptiMoM and Assad studies were counterbalanced by a decreased risk in the Sullivan reanalysis

Two studies provided data for the impact of fortifier type on PDA, and our secondary outcome feeds withheld for $>24$ hours (FW24). Figure 2 shows the CMDF group had a higher risk of $P D A(R R=1.6$; $\mathrm{P}=0.009)$ and FW24 (RR=3.4; $\mathrm{P}=0.0001)$.

\section{A meta-analysis of mortality/morbidity indices}

In OptiMoM, those fed CMDF had a large, near $40 \%$ increase in the risk of a positive mortality/morbidity index compared to the HMDF group. $(P=0.07)$ - see table 2. Because of the poten- 
Figure 1: meta-analyses for NEC, ROP, BPD, Death, LOS; all 3 studies contributed

Figure 1a NEC

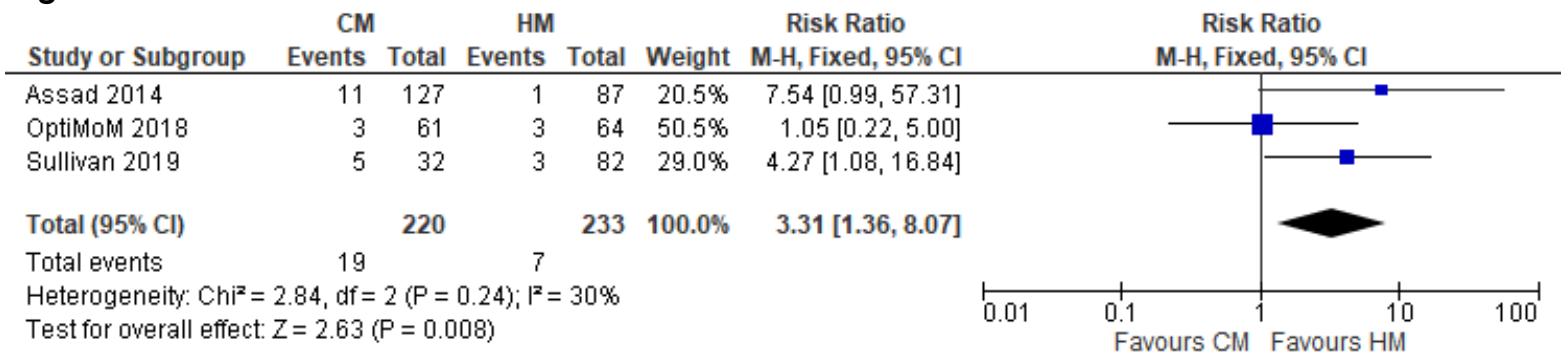

Figure $1 b$ ROP

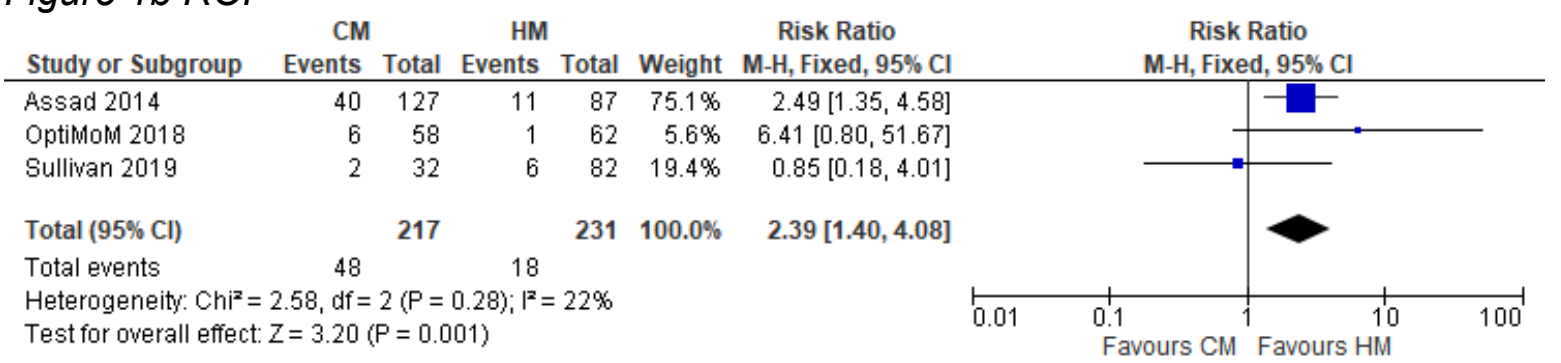

Figure 1c BPD

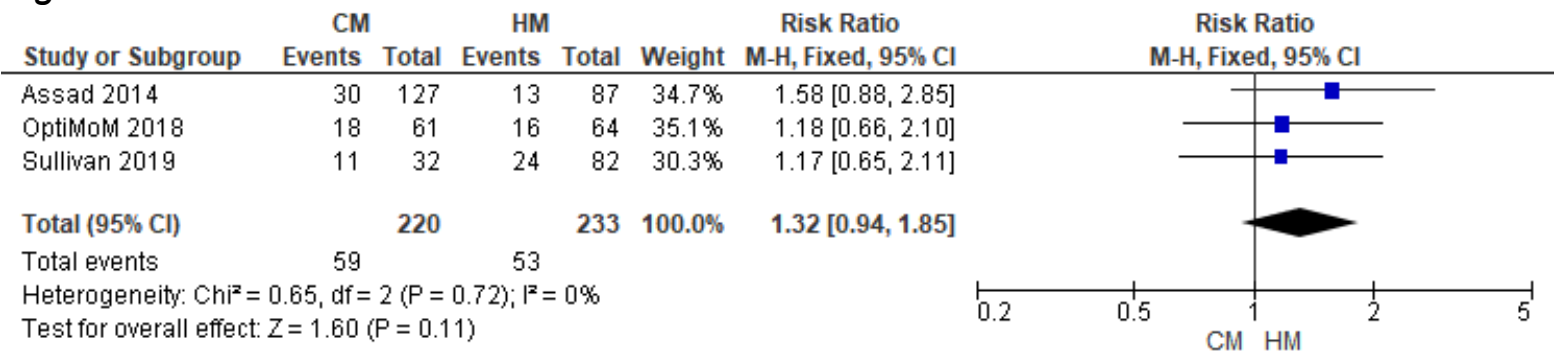

Figure 1d Death

\begin{tabular}{|c|c|c|c|c|c|c|c|c|c|c|}
\hline \multirow[b]{2}{*}{ Study or Subgroup } & \multicolumn{2}{|c|}{ Cows Milk } & \multicolumn{2}{|c|}{ Human Milk } & \multirow[b]{2}{*}{ Weight } & \multirow{2}{*}{$\begin{array}{c}\text { Risk Ratio } \\
\text { M-H, Fixed, } 95 \% \mathrm{Cl}\end{array}$} & \multirow{2}{*}{\multicolumn{3}{|c|}{$\begin{array}{c}\text { Risk Ratio } \\
\text { M-H, Fixed, } 95 \% \mathrm{Cl}\end{array}$}} & \\
\hline & Events & Total & Events & Total & & & & & & \\
\hline Assad 2014 & 3 & 127 & 1 & 87 & $20.5 \%$ & $2.06[0.22,19.43]$ & & & & \\
\hline OptiMom 2018 & 4 & 61 & 3 & 64 & $50.5 \%$ & $1.40[0.33,6.00]$ & & & & \\
\hline Sullivan 2019 & 4 & 32 & 3 & 82 & $29.0 \%$ & $3.42[0.81,14.42]$ & & & & \\
\hline Total $(95 \% \mathrm{Cl})$ & & 220 & & 233 & $100.0 \%$ & $2.12[0.85,5.31]$ & & & & \\
\hline Total events & 11 & & 7 & & & & & & & \\
\hline $\begin{array}{l}\text { Heterogeneity: } \mathrm{Chi}^{2} \\
\text { Test for overall effec }\end{array}$ & $\begin{array}{l}0.74, d f= \\
Z=1.60\end{array}$ & $\begin{array}{l}2(P= \\
P=0.1\end{array}$ & $\begin{array}{l}0.69) ;\left.\right|^{2}= \\
\text { 1) }\end{array}$ & & & & 0.05 & 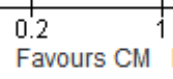 & $\begin{array}{r}1 \\
5 \\
\text { Favours HM }\end{array}$ & 20 \\
\hline
\end{tabular}

Figure 1e Late onset sepsis (LOS)

Cows Milk Human Milk

Study or Subgroup Events Total Events Total Weight M-H, Fixed, 95\% Cl

\begin{tabular}{lrrrrrr}
\hline Assad 2014 & 20 & 127 & 11 & 87 & $40.7 \%$ & $1.25[0.63,2.47]$ \\
OptiMoM 2018 & 14 & 61 & 8 & 64 & $24.3 \%$ & $1.84[0.83,4.06]$ \\
Sullivan 2019 & 5 & 32 & 20 & 82 & $35.0 \%$ & $0.64[0.26,1.56]$ \\
& & & & & & \\
Total $(95 \% \mathrm{Cl})$ & 39 & & 39 & & & \\
Total events & 39 & & $390.0 \%$ & $1.18[0.76,1.83]$
\end{tabular}

Heterogeneity. $\mathrm{Ch}^{2}=3.02, \mathrm{df}=2(\mathrm{P}=0.22) ; \mathrm{I}^{2}=34 \%$

Test for overall effect: $Z=0.73(P=0.47)$

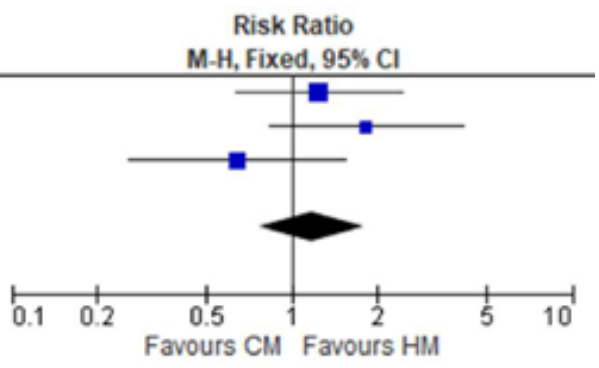


Figure 2: meta-analyses where data on 2 out of the 3 studies were available. Outcomes were PDA, and the secondary outcome: feeds withheld $>24$ hours

Figure 2a PDA

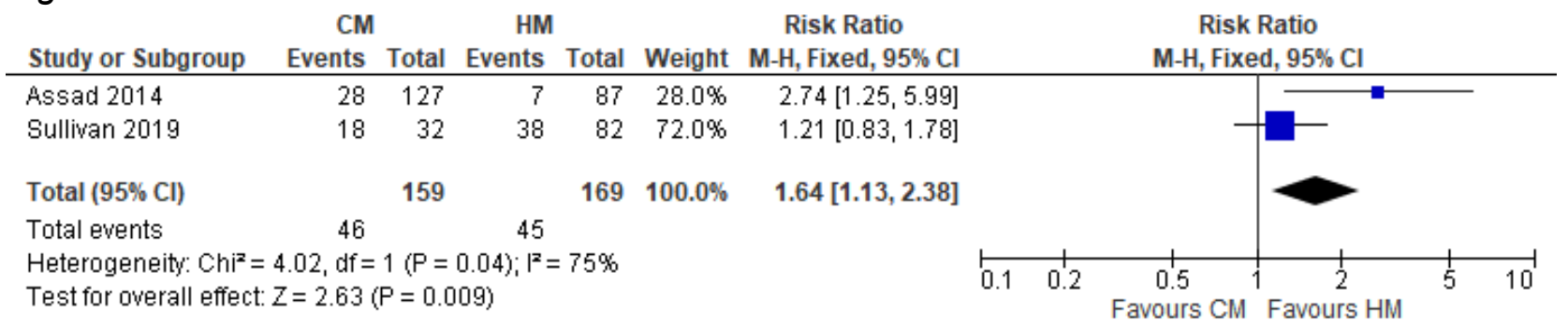

Figure 2c Feed withheld $>24$ hours

\begin{tabular}{|c|c|c|c|c|c|c|c|c|c|c|}
\hline Study or Subgroup & \multicolumn{2}{|l|}{$\mathrm{CM}$} & \multicolumn{2}{|l|}{ HM } & Weight & $\begin{array}{c}\text { Risk Ratio } \\
\text { M-H, Fixed, } 95 \% \mathrm{Cl} \\
\end{array}$ & \multicolumn{3}{|c|}{$\begin{array}{c}\text { Risk Ratio } \\
\text { M-H, Fixed, } 95 \% \mathrm{Cl}\end{array}$} & \\
\hline Assad 2014 & 43 & 127 & 5 & 87 & $40.3 \%$ & $5.89[2.43,14.27]$ & & & - & \\
\hline OptiMom 2018 & 14 & 61 & 9 & 64 & $59.7 \%$ & $1.63[0.76,3.49]$ & & & & \\
\hline Total $(95 \% \mathrm{Cl})$ & & 188 & & 151 & $100.0 \%$ & $3.35[1.90,5.92]$ & & & & \\
\hline Total events & 57 & & 14 & & & & & & & \\
\hline $\begin{array}{l}\text { Heterogeneity: } \mathrm{Chi}^{2}= \\
\text { Test for overall effect }\end{array}$ & $\begin{array}{l}5.00, \mathrm{df}= \\
Z=4.16(\end{array}$ & $\begin{array}{l}1(P=1 \\
(P<0.0\end{array}$ & $\begin{array}{l}0.03) ; 1^{2}= \\
0001)\end{array}$ & $=80 \%$ & & & 0.01 & ${ }_{\text {Favours } \mathrm{CM}}^{1}$ & $\begin{array}{r}10 \\
\text { Favours HM }\end{array}$ & 100 \\
\hline
\end{tabular}

Figure 3: Meta-analysis of morbidity indices in the OptiMoM, Assad, and Sullivan studies, A positive index is defined as one or more of death, sepsis, NEC, ROP and BPD. The index was based on the one published in the OptiMoM corrigendum (27), and equivalent indices (based on the same 5 outcomes) were derived from raw data in the Sullivan and Assad reanalysis.

\begin{tabular}{|c|c|c|c|c|c|c|c|c|}
\hline Study or Subgroup & \multicolumn{2}{|c|}{$\mathrm{CM}$} & \multicolumn{2}{|l|}{ HM } & \multicolumn{2}{|r|}{ Risk Ratio } & \multicolumn{2}{|c|}{$\begin{array}{c}\text { Risk Ratio } \\
\text { M-H, Fixed, } 95 \% \text { Cl }\end{array}$} \\
\hline Assad 2014 & 60 & 127 & 25 & 87 & $40.1 \%$ & $1.64[1.13,2.40]$ & & $=$ \\
\hline OptiMoM 2018 & 30 & 61 & 23 & 64 & $30.3 \%$ & $1.37[0.90,2.07]$ & & \\
\hline Sullivan 2019 & 16 & 32 & 39 & 82 & $29.6 \%$ & $1.05[0.69,1.59]$ & & \\
\hline Total $(95 \% \mathrm{Cl})$ & & 220 & & 233 & $100.0 \%$ & $1.39[1.10,1.75]$ & & \\
\hline Total events & 106 & & 87 & & & & & \\
\hline $\begin{array}{l}\text { Heterogeneity: } \mathrm{Ch}^{2} \\
\text { Test for overall effec }\end{array}$ & $\begin{array}{l}2.49, d f= \\
Z=2.74\end{array}$ & $\begin{array}{l}2(P= \\
(P=0.0\end{array}$ & $\begin{array}{l}0.29) ; 1^{2}= \\
06)\end{array}$ & $=20 \%$ & & & ${ }_{\text {Favours }}^{0.5}{ }^{1}$ & Favours $\stackrel{2}{\mathrm{HM}}$ \\
\hline
\end{tabular}

tial clinical importance of this, we derived the same index for the Assad and Sullivan studies based on the raw data (one or more of NEC, sepsis, BPD, ROP, or death). Fig. 3, shows a significant $40 \%$ higher risk of a positive mortality/morbidity index in the CMDF group $(R R=1.4 ; P=0.006)$ when the three studies were combined in a meta-analysis.

\section{Discussion}

We found strong evidence that using CMDF, even with a $100 \%$ $\mathrm{HM}$ base diet, was associated with an increased risk of major neonatal morbidities. This finding is consistent with previous studies, including RCTs, cohort studies, systematic reviews, and metaanalyses, which indicated that feeding CM based products (PTF and $\mathrm{CMDF}$ ) was associated with increased risk of multiple major neonatal morbidities (1-12).
In each of the three studies, analyzed separately, individual adverse outcomes were increased in the CMDF groups. When the studies were combined in meta-analyses, a clearer pattern of increased risk emerged for CMDF, most strongly expressed for NEC, ROP, PDA and FW24, and a dichotomous mortality/morbidity index based on death, late-onset sepsis, NEC, ROP or BPD.

One factor that may have an important bearing on these findings is that even with a $100 \% \mathrm{HM}$ base diet, CM protein consumption of VLBW infants is still unexpectedly high. The current guideline that trades use of PTF for the use of DM is focussed on maximizing HM intake to improve outcomes. Yet, this practice also increases the use of fortifier - most commonly CMDF. We estimate a VLBW baby targeted to receive $4 \mathrm{~g}$ protein $/ \mathrm{kg} /$ day would obtain only $50 \%$ of this from $160 \mathrm{ml} / \mathrm{kg}$ per day of mother's preterm milk 
Table 1: Baseline characteristics comparing infants fed a human milk (HMDF) vs cow's milk (CMDF) derived fortifier

\begin{tabular}{|c|c|c|}
\hline Parameter & HMDF & CMDF \\
\hline \multicolumn{3}{|c|}{ OptiMoM trial } \\
\hline Number of subjects & 64 & 63 \\
\hline Sex(female) & $39 / 64(61 \%)$ & $34 / 63(54 \%)$ \\
\hline Race (non-European) & $37 / 64(59 \%)$ & $44 / 63(71 \%)$ \\
\hline Antenatal steroids & $56 / 64(88 \%)$ & $56 / 63(89 \%)$ \\
\hline Apgar at $5 \mathrm{~min}$ & $7.4(\mathrm{SD} 2.1)$ & $7.3(\mathrm{SD} 2.3)$ \\
\hline Gestation (weeks) & $27.9(\mathrm{SD} 2.7)$ & $27.5(\mathrm{SD} 2.3)$ \\
\hline Birthweight (grams) & 887 (SD 208) & 889 (SD 196) \\
\hline SGA at birth & $13 / 64(20 \%)$ & $16 / 63(25 \%)$ \\
\hline \multicolumn{3}{|c|}{ Sullivan trial re-analysis } \\
\hline Number of subjects & 82 & 32 \\
\hline Sex (female) & $47 / 82(57.3 \%)$ & $15 / 32(46.9 \%)$ \\
\hline Race (black) & $16 / 82(19.5 \%)$ & $3 / 32(9.4 \%)$ \\
\hline Antenatal steroids & $15 / 82(18.3 \%)$ & $6 / 32(18.8 \%)$ \\
\hline APGAR $<7$ & $8 / 82(9.8 \%)$ & $6 / 32(18.8 \%)$ \\
\hline Gestation (weeks) & $27.3 \pm 2.2$ & $27.1 \pm 1.8$ \\
\hline Birthweight (grams) & $937 \pm 199$ & $938 \pm 190$ \\
\hline SGA at birth & $10 / 82(12.2 \%)$ & $3 / 32(9.4 \%)$ \\
\hline \multicolumn{3}{|c|}{ Assad study re-analysis } \\
\hline Number of subjects & 87 & 127 \\
\hline Sex (female) & $34 / 87(39 \%)$ & $64 / 127(50 \%)$ \\
\hline Race (black) & $53(61 \%)$ & $85 / 127(67 \%)$ \\
\hline Gestation (weeks) & $27.7(\mathrm{SD} 2.7)$ & $28.3(\mathrm{SD} 2.8)$ \\
\hline
\end{tabular}

Statistical tests used: $t$-test for quantitative variables and chi-square/Fisher's exact test for categorical variables; all comparisons between groups were non-significant $(p>0.05)$

NEONATOLOGY TODAY is interested in publishing manuscripts from Neonatologists, Fellows, NNPs and those involved in caring for neonates on case studies, research results, hospital news, meeting announcements, and other pertinent topics.

Please submit your manuscript to: LomaLindaPublishingCompany@gmail.com 
Table 2: Outcomes comparing infants fed a human milk (HMDF) vs cow's milk (CMDF) derived fortifier

\begin{tabular}{|c|c|c|c|c|}
\hline Parameter & HMDF & CMDF & $\mathbf{R R}^{1}$ & P-value ${ }^{2}$ \\
\hline Total number subjects (453) & 233 & 220 & & \\
\hline \multicolumn{5}{|c|}{ OptiMOM trial } \\
\hline Number of subjects & 64 & 61 & & \\
\hline ROP (severe) & $1 / 62(1.6 \%)$ & $6 / 59 ; 10 . \%)$ & 6.4 & 0.04 \\
\hline Late onset sepsis & $8 / 64(13 \%)$ & $14 / 61(23 \%)$ & 1.8 & $0.07^{3}$ \\
\hline Death & $3 / 64(4.7 \%)$ & $4(6.6 \%)$ & 1.4 & 0.65 \\
\hline BPD & $16 / 64(25 \%)$ & $18 / 61(30 \%)$ & 1.2 & $0.73^{3}$ \\
\hline NEC (Bells stage II or greater) & $3 / 64(4.7 \%)$ & $3 / 61(4.9 \%)$ & 1.0 & 0.95 \\
\hline NEC all stages & $3 / 64(4.7 \%)$ & $6 / 61(9.8 \%)$ & 2.1 & 0.27 \\
\hline Feeds withheld 12h (FW12h) & $17 / 64(27 \%)$ & $20 / 61(33 \%)$ & 1.2 & $0.34^{3}$ \\
\hline Feeds withheld 24h (FW24h) & $9 / 64(14 \%)$ & $14 / 61(23 \%)$ & 1.6 & $0.19^{3}$ \\
\hline Positive morbidity index ${ }^{4}$ & $23 / 64(36 \%)$ & $30 / 61(49 \%)$ & 1.4 & $0.07^{3}$ \\
\hline \multicolumn{5}{|c|}{ Sullivan $R C T-$ subgroup reanalysis } \\
\hline Number of subjects & 82 & 32 & & \\
\hline NEC (Bells Stage II or greater) & $3 / 82(3.7 \%)$ & $5(15.6 \%)$ & 4.2 & 0.04 \\
\hline NEC surgery or death & $3 / 82(3.7 \%)$ & $6 / 32(18.8 \%)$ & 5.1 & 0.01 \\
\hline Death only & $3 / 82(3.7 \%)$ & $4 / 32(12.5 \%)$ & 3.4 & 0.10 \\
\hline Proven sepsis & $20 / 84(24.4 \%)$ & $5 / 32(15.6 \%)$ & 0.6 & 0.45 \\
\hline BPD & $24 / 84(29.3 \%)$ & $11 / 32(34.4 \%)$ & 1.2 & 0.60 \\
\hline ROP (grade 3 or 4 ) & $6 / 84(7.3 \%)$ & $2 / 32(6.3 \%)$ & 0.9 & 1.0 \\
\hline \multicolumn{5}{|c|}{ Assad study - subgroup group reanalysis } \\
\hline Number of subjects & 87 & 127 & & \\
\hline NEC (Bells stage II or greater) & $1 / 87 \quad(1.1 \%)$ & $11 / 127(8.7 \%)$ & 7.5 & 0.02 \\
\hline ROP & $11 / 87(14 \%)$ & $40 / 127(32 \%)$ & 2.5 & 0.001 \\
\hline BPD & $13 / 87(15 \%)$ & $30 / 127(24 \%)$ & 1.6 & 0.20 \\
\hline $\mathrm{PDA}^{5}$ & $7 / 87 \quad(8 \%)$ & $28 / 127(22 \%)$ & 2.7 & 0.007 \\
\hline Feeds withheld 24h (FW24) & $5 / 87 \quad(6 \%)$ & $43 / 127(34 \%)$ & 5.9 & 0.001 \\
\hline Late onset sepsis & $11 / 87(13 \%)$ & $20 / 127(16 \%)$ & 1.3 & 0.66 \\
\hline
\end{tabular}

${ }^{1} R R=$ relative risk of adverse outcome in the CMDF group

${ }^{2}$ chi-square/Fisher's exact test

${ }^{3}$ adjusted $P$ value based on multivariate logistic regression model

${ }^{4}$ Positive mortality/morbidity indxex = one or more of: ROP, sepsis, death, BPD, NEC stage II or greater ${ }^{5}$ Data on PDA collected in original database but not published in the original Assad Study

Death

\begin{tabular}{|ll|l}
$1 / 87$ & $(1.1 \%)$ & $3 / 127$ \\
\hline
\end{tabular}

$(2.4 \%)$

2.2

0.89 
of average composition (28) and only around $40 \%$ of this intake from $160 \mathrm{ml} / \mathrm{kg}$ of typical DM. The rest of this protein requirement must be from CMDF. Thus, babies fed $\mathrm{HM}$ as recommended may derive more protein from CMDF than $\mathrm{HM}$, perhaps accounting for the apparently large adverse impact of CMDF.

\section{"We found strong evidence that using CMDF, even with a $100 \%$ HM base diet, was associated with an increased risk of major neonatal morbidities."}

An aspect of note was the variation between studies in the diversity of outcomes related to the use of CMDF. The OptiMoM trial showed that compared to the HMDF group, those fed CMDF had a strong trend to a near $40 \%$ increase in the proportion of subjects with a positive morbidity index, based on diverse morbidities. The Assad reanalysis illustrated this diversity of adverse outcomes with CMDF even more strikingly. Nevertheless, conversely, in the Sullivan reanalysis, the dominant effect of CMDF was in relation to NEC, NEC surgery, and death: whereas sepsis, ROP and BPD appeared unaffected. A likely possibility is that this simply reflects random variation in small individual studies, and hence justifies our approach in using meta-analyses to combine the data,

One finding in our trial that deserves attention is the hitherto unexplained higher risk of patent ductus in those fed CMDF. This was observed here in a meta-analysis of two of our three studies but has been previously reported by Hair and co-workers7, who found a highly significant increase in the risk of PDA in those exposed to $\mathrm{CM}$ products versus an exclusive human milk base diet. This requires further investigation.

Many studies lack the power to detect significant effects on the outcomes studied. To increase power, dichotomous morbidity indices are often used where having one or more of a group of adverse outcomes is treated as a positive result. In the OptiMoM trial, a mortality/morbidity index was used (one or more of death, NEC, sepsis, ROP, or BPD); and the CMDF group had a $40 \%$ increased risk of a positive index, based on the most serious common outcomes in neonatal care. Yet because $P=0.07$, the authors rejected this as "not significant" (27). However, Amrhein et al. in a seminal 2019 Nature paper (29), with 800 signatories, argue that $P$ values have been misused, that $P$ should not be a dichotomous variable with an arbitrary cut off value such as 0.05 and cannot be used to determine that findings are 'not significant,' particularly with large RRs, since this may result in incorrect rejection of key findings. Because of the potential clinical importance here, we conducted a meta-analysis of this same mortality/morbidity index for all three of our studies ( $n=453$ versus 125 in OptiMoM alone: fig 3 ). The increased risk of having a positive index in the CMDF group remained at $40 \%$, but now, in this more powered analysis, $\mathrm{P}=0.006$.

Our secondary outcome and a primary one in OptiMoM (9) was the interruption of feeds - a major problem that increases the need for PN and its associated morbidity. In OptiMoM, feed interruption was measured as feeds withheld for 12 hours (FW12) but also as FW24. The increased risk for feed interruption for 12 and 24 hours in OptiMoM in the CMDF group was sizeable at $22 \%$ and $63 \%$, respectively, but rejected as not significant. In OptiMoM, CM was introduced late (see below), which could have reduced the power of the study. Since FW24 was measured in both OptiMoM and Assad, a more robust meta-analysis was possible, showing a 3.4-fold increase in FW24 $(P=0.0001)$ with the use of CMDF (see figure 2); an effect size potentially of considerable clinical significance.

\section{Limitations}

Our study is not a conventional systematic review or meta-analysis of published evidence and could not be conducted according to conventional guidelines. For 2 of our three studies (Sullivan and Assad), these could not be searched since they did not preexist but were created from subgroup analyses of the raw data from the original studies; and we accept there might be further, suitable raw datasets that could be correspondingly analyzed.

Two of our studies were subgroup analyses, theoretically more prone to chance imbalances between groups; greater morbidity in the CMDF group might simply have reflected chance generation a higher risk population. The evidence is strongly against that. Our studies, conducted in different years and centers, consistently showed greater morbidity in those fed CDMF. Baseline risk factors were well balanced between groups in each study. Also, much evidence links CM exposure to the same adverse outcomes shown here.

The NEC incidence amongst our three studies deserves some comment. The most modern of the three studies (OptiMoM) showed a particularly low incidence of NEC with no difference between randomized groups. Whilst we agree that future studies will resolve if this is an exceptional finding or not, we would note that the adverse effects of CM may be ameliorated by delaying its introduction into the diet (though with corresponding downsides for growth and requirement for $\mathrm{PN}$ ). In the OptiMoM trial, the mean age of introduction of CM was late at day 17 (9), compared to the possibility of receiving $\mathrm{CM}$ in the early part of the first week in the Sullivan trial (1). The incidence of NEC in the Sullivan reanalysis was higher than commonly seen today, even in very small infants. However, the key point is that despite the heterogeneity in feeding practice, age of study, etc., our meta-analysis indicates a major increase in NEC in those assigned CMDF vs. HMDF: $8.6 \%$ incidence vs. $3.0 \%$ (derived from the data in figure 1 ).

\section{"Our secondary outcome and a primary one in OptiMoM (9) was the interruption of feeds - a major problem that increases the need for PN and its associated morbidity."}

Our study does not address the use of liquid CMDF used now in the US, though powder-based fortifiers are used in many countries and the three studies here. Whether liquid fortifiers by displacing about $1 / 6$ th of the MOM or DM volume with a fortifier could further increase any adverse impact of CMDF has received little attention. It would be hard to explore if HMDF, which is also a liquid, commercially derived from pasteurized DM, could have any measurable impact on the outcome by displacing a significant volume of MOM when the available volume is high. However, overall, compared to VLBW infants exposed to CM products, those fed on an EHM diet including HMDF, studied by us here and by other groups $(1-3,7,10)$ have in general significantly lower morbidities

Finally, our findings apply to intact protein fortifiers in widespread international use. We do not consider here partially or extensively hydrolyzed fortifiers, now often used in the US (30-32). Such fortifiers have been compared with each other with some differences but not with HMDF and not studied for the broad range of morbidities reported to be differentially affected by CM vs. HM exposure. 
Moreover, the hypothesis that using hydrolyzed CM protein in feeds for preterm infants would overcome the adverse effects of intact CM products is unproven, and not supported conceptually by a recent Cochrane review (33), albeit focussed on hydrolyzed formulas rather than fortifiers.

\section{Conclusion}

Current recommendations to maximize human milk intake in preterm infants have been enthusiastically implemented internationally, but without adequate research on the impact of the CM component of the diet with this new regime. This now needs scientific attention. We have identified three studies all with some form of experimental design that show individually, and collectively in meta-analyses, that VLBW preterm infants fed CMDF with an otherwise $100 \%$ human milk base diet had a significant increase in major morbidities some of which may reduce survival or have significant adverse post-neonatal effects. This burden of morbidity, indicated by the findings, provides evidence that the benefits of an $\mathrm{HM}$ base diet, might be in part counteracted by multiple adverse outcomes relating to the use of CMDF- and this needs further research attention. Our study demonstrated a significantly lower burden of morbidity with HMDF. Finally, the use of CMDF is designed to meet nutrient needs, yet our study emphasizes further the increasingly recognized potential importance of non-nutritional impacts of diet on clinical course and health outcomes in this highrisk population.

"We have identified three studies all with some form of experimental design that show individually, and collectively in metaanalyses, that VLBW preterm infants fed CMDF with an otherwise $100 \%$ human milk base diet had a significant increase in major morbidities some of which may reduce survival or have significant adverse post-neonatal effects. "

References:

1. Sullivan S, Schanler R, Kim J et al. An Exclusively Human Milk-Based Diet Is Associated with a Lower Rate of Necrotizing Enterocolitis than a Diet of Human Milk and Bovine Milk-Based Products. J Pediatr. 2010;156(4):562567.e1. doi:10.1016/j.jpeds.2009.10.040

2. Cristofalo E, Schanler R, Blanco $C$ et al. Randomized Trial of Exclusive Human Milk versus Preterm Formula Diets in Extremely Premature Infants. J Pediatr. 2013;163(6):15921595.e1. doi:10.1016/j.jpeds.2013.07.011

3. Quigley $M$, Embleton $N$, McGuire $W$. Formula versus donor breast milk for feeding preterm or low birth weight infants. Cochrane Database of Systematic Reviews. 2018. doi:10.1002/14651858.cd002971.pub4

4. Lucas A, Cole T. Breast milk and neonatal necrotising enterocolitis. The Lancet. 1990;336(8730-8731):1519-1523. doi:10.1016/0140-6736(90)93304-8

5. Narayanan I, Prakash K, Murthy NS, Gujral VV. Randomised controlled trial of effect of raw and holder pasteurised human milk and of formula supplements on incidence of neonatal infection. Lancet 1984;ii:1111-3
6. de Silva $A$. Does human milk reduce infection rates in preterm infants? A systematic review. Archives of Disease in Childhood - Fetal and Neonatal Edition. 2004;89(6):F509-F513. doi:10.1136/adc.2003.045682

7. Hair A, Rechtman D, Lee M, Niklas V. Beyond Necrotizing Enterocolitis: Other Clinical Advantages of an Exclusive Human Milk Diet. Breastfeeding Medicine. 2018;13(6):408411. doi:10.1089/bfm.2017.0192

8. Abrams S, Schanler R, Lee M, Rechtman D. Greater Mortality and Morbidity in Extremely Preterm Infants Fed a Diet Containing Cow Milk Protein Products. Breastfeeding Medicine. 2014;9(6):281-285. doi:10.1089/bfm.2014.0024

9. O'Connor D, Kiss A, Tomlinson $C$ et al. Nutrient enrichment of human milk with human and bovine milk-based fortifiers for infants born weighing <1250 g: a randomized clinical trial. Am J Clin Nutr. 2018. doi:10.1093/ajcn/nqy067

10. Assad M, Elliott M, Abraham J. Decreased cost and improved feeding tolerance in VLBW infants fed an exclusive human milk diet. Journal of Perinatology. 2015;36(3):216-220. doi:10.1038/jp.2015.168

11. Manzoni $P$, Stolfi $I$, Pedicino $R$ et al. Human milk feeding prevents retinopathy of prematurity (ROP) in preterm VLBW neonates. Early Hum Dev. 2013;89:S64-S68. doi:10.1016/ s0378-3782(13)70019-7

12. Colacci M, Murthy K, DeRegnier R, Khan J, Robinson D. Growth and Development in Extremely Low Birth Weight Infants After the Introduction of Exclusive Human Milk Feedings. Am J Perinatol. 2017;34(02):130-137. doi:10.1055/s-0036-1584520

13. Anderson J, Johnstone B, Remley D. Breast-feeding and cognitive development: a meta-analysis. Am J Clin Nutr. 1999;70(4):525-535. doi:10.1093/ajcn/70.4.525

14. Lucas A, Morley R, Cole T, Lister G, Leeson-Payne C. Breast milk and subsequent intelligence quotient in children born preterm. International Journal of Gynecology \& Obstetrics. 1992;39(2):164-164. doi:10.1016/0020-7292(92)90963-j

15. Singhal $A$, Cole $T$, Lucas $A$. Early nutrition in preterm infants and later blood pressure: two cohorts after randomised trials. The Lancet. 2001;357(9254):413-419. doi:10.1016/ s0140-6736(00)04004-6

16. Singhal A, Fewtrell M, Cole T, Lucas A. Low nutrient intake and early growth for later insulin resistance in adolescents born preterm. The Lancet. 2003;361(9363):1089-1097. doi:10.1016/s0140-6736(03)12895-4

17. Singhal $A$, Cole $T$, Fewtrell $M$, Lucas $A$. Breastmilk feeding and lipoprotein profile in adolescents born preterm: follow-up of a prospective randomised study. The Lancet. 2004;363(9421):1571-1578. doi:10.1016/s01406736(04)16198-9

18. Fewtrell $M$, Williams $J$, Singhal $A$, Murgatroyd $P$, Fuller $N$, Lucas A. Early diet and peak bone mass: 20 year follow-up of a randomized trial of early diet in infants born preterm. Bone. 2009;45(1):142-149. doi:10.1016/j.bone.2009.03.657

19. Lucas A, Brooke O, Morley R, Cole T, Bamford M. Early diet of preterm infants and development of allergic or atopic disease: randomised prospective study. BMJ. 1990;300(6728):837-840. doi:10.1136/bmj.300.6728.837

20. Lewandowski $A$, Lamata $P$, Francis $J$ et al. Breast Milk Consumption in Preterm Neonates and Cardiac Shape in Adulthood. Pediatrics. 2016;138(1):e20160050-e20160050. doi:10.1542/peds.2016-0050

21. AAP Section on Breastfeeding. Breastfeeding and the Use of Human Milk. Pediatrics. 2012;129(3):e827-e841. doi:10.1542/peds.2011-3552

22. ESPGHAN Committee on Nutrition, Arslanoglu S, Corpeleijn $W$, Moro $G$ et al. Donor human milk for preterm infants: current evidence and research directions. J Pediatr 
Gastroenterol Nutr. 2013;57(4):535-542. doi:10.1097/ mpg.0b013e3182a3af0a

23. Polberger S, Raiha NC, Juvonen P, Moro GE, Minoli I, Warm A Individualized protein fortification of human milk for preterm infants: comparison of ultrafiltrated human milk protein and a bovine whey fortifier. J Pediatr Gastroenterol Nutr 1999;29(3):332-8.

24. Hagelberg S, Lindblad BS, Persson B. Amino acid levels in the critically ill preterm infant given mother's milk fortified with protein from human or cow's milk. Acta Paediatr Scand 1990;79(12):1163

25. Lucas A. Boscardin J, Abrams SA.Preterm infants fed cows' milk-derived fortifier had averse outcomes despite a base diet of only mother's own milk. Breast Feeding Medicine, 2020; 15(5): 1-7. DOI 10.1089/bfm.2019.0133

26. Borenstein, M. Hedges, L., \& Rothstein, H. (2007). MetaAnalysis: Fixed effect vs. random effects. (page 30) Retrieved from www.Meta-Analysis.com

27. Corrigendum for O'Connor et al. Nutrient enrichment of human milk with human and bovine milk-based fortifiers for infants born weighing < $1250 \mathrm{~g}$ : a randomized clinical trial. Am J Clin Nutr 2018;108:108-16. Am J Clin Nutr. 2019;110:529.

28. Boyce C, Watson M, Lazidis G, Reeve S, Dods K, Simmer K et al. Preterm human milk composition: a systematic literature review. British Journal of Nutrition. 2016;116(6):1033-1045.

29. Amrhein V, Greenland S, McShane B. Scientists rise up against statistical significance. Nature. 2019;567(7748):3057.

30. Kim J, Chan G, Schanler $R$ et al. Growth and Tolerance of Preterm Infants Fed a New Extensively Hydrolyzed Liquid Human Milk Fortifier. J Pediatr Gastroenterol Nutr. 2015;61(6):665-671. doi:10.1097/mpg.0000000000001010

31. Monga R, Sampath V, Ehrhart B, Kumar N. Prospective Comparison of Enfamil and Similac Liquid Human Milk Fortifier on Clinical Outcomes in Premature Infants. Am J Perinatol. 2017;34(14):1411-1416. doi:10.1055/s-0037-1603940

32. Lainwala S, Kosyakova N, Spizzoucco A, Herson V, Brownell E. Clinical and nutritional outcomes of two liquid human milk fortifiers for premature infants. J Neonatal Perinatal Med. 2017;10(4):393-401. doi:10.3233/npm-16164

33. Ng DHC, Klassen JRL, Embleton ND, McGuire W. Protein hydrolysate versus standard

formula for preterm infants. Cochrane Database of Systematic Reviews 2019, Issue 7. art.no.: CD012412. DOI: 10.1002/14651858.CD012412.pub3

\section{Acknowledgments}

We acknowledge the kind permission of Dr. Melinda Elliott for allowing us to reanalyze her raw data for the study by Assad et al. and for painstakingly retrieving further raw data from the patient records

\section{Author Contributions}

Professor Alan Lucas conceived and initiated the study; analyzed raw data; and wrote the paper

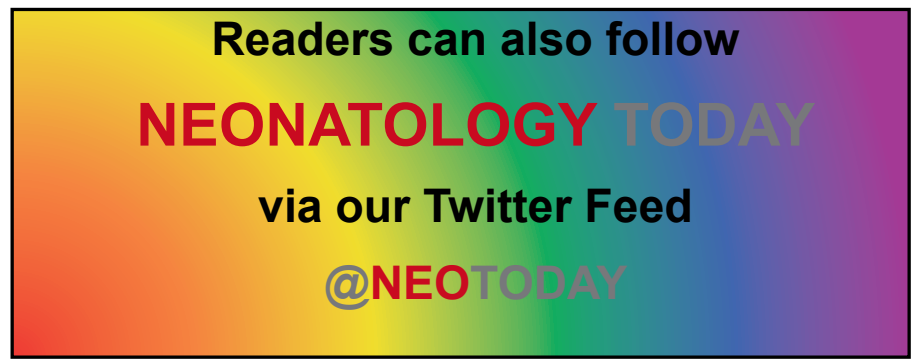

Dr Maushumi Assad provided her raw data (Assad study) and assisted us with the understanding of her database, She read and commented constructively on the manuscript

Professor Jan Sherman was our advisor on evidence-based medicine. She performed all the meta-analyses and provided the data for figures. She read and advised on the manuscript.

Dr. John Boscardin was our statistician who advised on: the statistical analysis, interpretation of data and data presentation

Professor Steven Abrams key collaborator involved in every aspect of the study and made major intellectual input.

Financial support: None:

Conflicts of Interest: Dr. Lucas has provided independent scientific advice to Philips, Prolacta, and Nestle.; Dr. Assad, none; Dr. Sherman, none; Dr. Boscardin, none. Dr. Abrams, none

NT

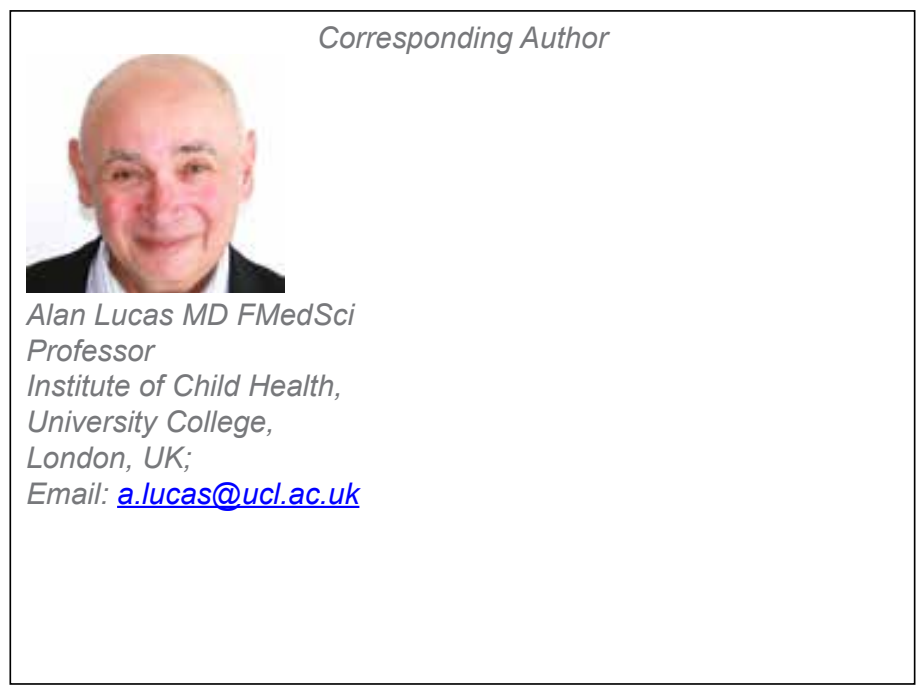




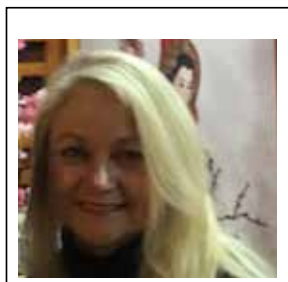

Jan Sherman PhD

Teaching Professor

School of Nursing

University of Missouri

Columbia, MO, USA

Adjunct Teaching Professor

School of Medicine

email: shermanj@missouri.edu

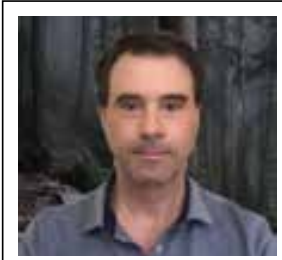

John Boscardin PhD

Professor

University of California

San Francisco, CA, USA

email:john.boscardin@gmail.com

Corresponding Author

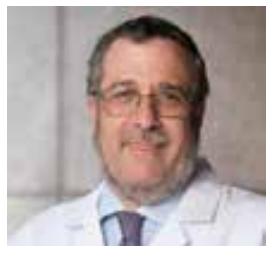

Steven Abrams MD

Professor

Department of Pediatrics

Dell Medical School

University of Texas,

Austin, TX, USA

email: sabrams@austin.utexas.edu
SHARED DECISION-MAKING PROTECTS

\section{MOTHERS + INFANTS}

\section{DURING COVID-19}

KEEPING MOTHERS + INFANTS TOGETHER

Means balancing...

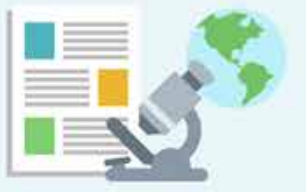

PARTNERSHIP

SHARED DECISION-MAKING

What is the best for this unique dyad?

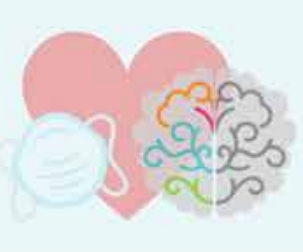

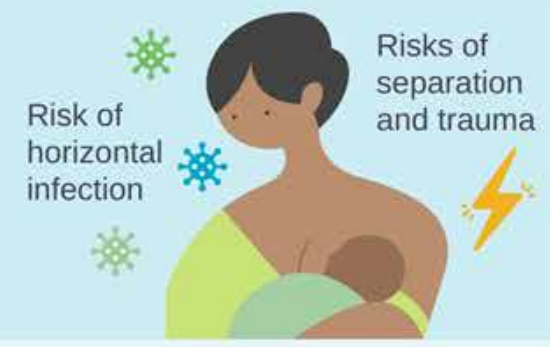

\section{EVIDENCE}

We encourage families and clinicians to remain diligent in learning up-to-date evidence.

\section{LONGITUDINAL DATA}

We need to understand more about outcomes for mothers and infants exposed to COVID-19, with special attention to:

- MENTAL HEALTH

- POSTPARTUM CARE DELIVERY

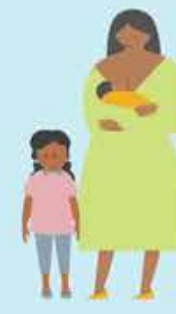

NEW DATA EMERGE DAILY

NANN AND NPA ENCOURAGE PERINATAL CARE PROVIDERS TO ENGAGE IN CANDID CONVERSATIONS WITH PREGNANT PARENTS PRIOR TO DELIVERY REGARDING RISKS, BENEFITS, LIMITATIONS, AND REALISTIC EXPECTATIONS.

Partnering for patient-centered care when it matters most.

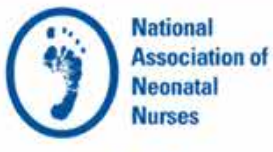

nann.org

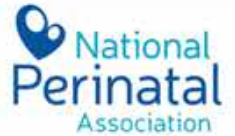

nationalperinatal.org 